\title{
POLYNOMIAL INSTANCES OF THE POSITIVE SEMIDEFINITE AND EUCLIDEAN DISTANCE MATRIX COMPLETION PROBLEMS*
}

\author{
MONIQUE LAURENT ${ }^{\dagger}$
}

Abstract. Given an undirected graph $G=(V, E)$ with node set $V=[1, n]$, a subset $S \subseteq V$, and a rational vector $a \in Q^{S \cup E}$, the positive semidefinite matrix completion problem consists of determining whether there exists a real symmetric $n \times n$ positive semidefinite matrix $X=\left(x_{i j}\right)$ satisfying $x_{i i}=a_{i}(i \in S)$ and $x_{i j}=a_{i j}(i j \in E)$. Similarly, the Euclidean distance matrix completion problem asks for the existence of a Euclidean distance matrix completing a partially defined given matrix. It is not known whether these problems belong to NP. We show here that they can be solved in polynomial time when restricted to the graphs having a fixed minimum fillin, the minimum fill-in of graph $G$ being the minimum number of edges needed to be added to $G$ in order to obtain a chordal graph. A simple combinatorial algorithm permits us to construct a completion in polynomial time in the chordal case. We also show that the completion problem is polynomially solvable for a class of graphs including wheels of fixed length (assuming all diagonal entries are specified). The running time of our algorithms is polynomially bounded in terms of $n$ and the bitlength of the input $a$. We also observe that the matrix completion problem can be solved in polynomial time in the real number model for the class of graphs containing no homeomorph of $K_{4}$.

Key words, positive semidefinite matrix, Euclidean distance matrix, matrix completion, chordal graph, minimum fill-in, order of a graph, polynomial algorithm, bit model, real number model

AMS subject classifications. 05C50, 15A48, 15A57, 90C25

PII. S0895479899352689

\section{Introduction.}

1.1. The matrix completion problem. This paper is concerned with the completion problem for positive semidefinite and Euclidean distance matrices. The positive semidefinite matrix completion problem $(\mathrm{P})$ is defined as follows:

Given a graph $G=(V, E)$, a subset $S \subseteq V$, and a rational vector $a \in \mathrm{Q}^{S \cup E}$, determine whether there exists a real matrix $X=\left(x_{i j}\right)_{i, j \in V}$ satisfying

$$
X \succeq 0 \text { and } x_{i i}=a_{i}(i \in S), x_{i j}=a_{i j}(i j \in E) .
$$

(The notation $X \succeq 0$ means that $X$ is a symmetric positive semidefinite matrix or, for short, a psd matrix.) In other words, problem (P) asks whether a partially specified matrix can be completed to a psd matrix, the terminology of graphs being used as a convenient tool for encoding the positions of the specified entries. When problem (P) has a positive answer, one says that $a$ is completable to a psd matrix; a matrix $X$ satisfying (1.1) is called a psd completion of $a$ and a positive definite (pd) completion when $X$ is positive definite. We let $\left(\mathrm{P}_{s}\right)$ denote problem $(\mathrm{P})$ when $S=V$, i.e., when all diagonal entries are specified. If one looks for a pd completion, then one can assume without loss of generality that all diagonal entries are specified (cf. Lemma 2.5 ); this is, however, not obviously so if one looks for a psd completion (although this can be shown to be true when restricting the problem to the class of chordal graphs; cf. the proof of Theorem 3.5).

* Received by the editors February 26, 1999; accepted for publication (in revised form) by L. El Ghaoui June 5, 2000; published electronically December 20, 2000.

http://www.siam.org/journals/simax/22-3/35268.html

†CWI, P.O. Box 94079, 1090 GB Amsterdam, The Netherlands (monique@cwi.nl). 
A matrix $Y=\left(y_{i j}\right)_{i, j=1}^{n}$ is called a Euclidean distance matrix (a distance matrix, for short) if there exist vectors $u_{1}, \ldots, u_{n} \in \mathbf{R}^{k}$ (for some $k \geq 1$ ) such that

$$
y_{i j}=\left\|u_{i}-u_{j}\right\|^{2} \text { for } i, j=1, \ldots, n .
$$

(Here, $\|u\|$ denotes the Euclidean norm of vector $u \in \mathbf{R}^{k}$.) A set of vectors $u_{i}$ satisfying (1.2) is called a realization of $Y$. Note that all diagonal entries of a distance matrix are equal to zero. The Euclidean distance matrix completion problem (D) is defined as follows:

Given a graph $G=(V, E)$ and a rational vector $d \in \mathrm{Q}^{E}$, determine whether there exists a real matrix $Y=\left(y_{i j}\right)_{i, j \in V}$ satisfying

$$
Y \text { is a distance matrix and } y_{i j}=d_{i j}(i j \in E) \text {. }
$$

Hence problem (D) asks whether a partially specified matrix can be completed to a distance matrix.

As will be recalled in section 2.3 , psd matrices and distance matrices are closely related and, thus, their associated completion problems can often be treated in an analogous manner. These matrix completion problems have many applications, e.g., to multidimensional scaling problems in statistics (cf. [29]), to the molecule conformation problem in chemistry (cf. [11], [18]), and to moment problems in analysis (cf. [5]).

1.2. An excursion to semidefinite programming. The psd matrix completion problem is obviously an instance of the general semidefinite programming feasibility problem $(\mathrm{F})$ :

Given integral $n \times n$ symmetric matrices $Q_{0}, Q_{1}, \ldots, Q_{m}$, determine whether there exist real numbers $z_{1}, \ldots, z_{m}$ satisfying

$$
Q_{0}+z_{1} Q_{1}+\cdots+z_{m} Q_{m} \succeq 0 .
$$

The complexity status of problem (F) is a fundamental open question in the theory of semidefinite programming; this is true for both the Turing machine model and the real number model, the two most popular models of computation used in complexity theory. In particular, it is not known whether there exists an algorithm solving $(F)$ whose running time is polynomial in the size $L$ of the data, that is, the total space needed to store the entries of the matrices $Q_{0}, \ldots, Q_{m}$.

The Turing machine model (also called rational number model, or bit model; cf. [13]) works on rational numbers and, more precisely, on their binary representations; in particular, the running time of an elementary operation $(+,-, \times, \div)$ depends on the length of the binary representations of the rational numbers involved. Hence, the size $L$ of the data of problem (F) in this model can be defined as $m n^{2} L_{0}$, where $L_{0}$ is the maximum number of bits needed to encode an entry of a matrix $Q_{i}$. On the other hand, the real number model (introduced in [10]) works with real numbers and it assumes that exact real arithmetic can be performed; in particular, an elementary operation $(+,-, \times, \div)$ between any two real numbers takes unit time. Hence, the size $L$ of the data of $(F)$ in this model is equal to $m n^{2}$.

Semidefinite programming (SDP) deals with the decision problem $(F)$ and its optimization version:

$$
\begin{array}{ll}
\max & c^{T} z \\
\text { subject to } & Q_{0}+z_{1} Q_{1}+\cdots+z_{m} Q_{m} \succeq 0,
\end{array}
$$


where $c \in \mathrm{Q}^{m}$. SDP can be seen as a generalization of linear programming (LP), obtained by replacing the nonnegativity constraints of the vector variable in LP by the semidefiniteness of the matrix variable in SDP. Information about SDP can be found in the handbook [42]; cf. also the survey [40] and [3], [17] with an emphasis on applications to discrete optimization.

A standard result in LP is that every feasible linear system $A x \leq b$ with rational coefficients has a solution whose size is polynomially bounded in terms of the size of $A$ and $b$ (cf. [38], Corollary 3.2b). This implies that the problem of testing the feasibility of an LP program belongs to NP in the bit model. (This fact is obvious for the real number model.) Moreover, any LP optimization problem can be solved in polynomial time in the bit model using the ellipsoid algorithm of Khachiyan [23] or the interior-point method of Karmarkar [22]; it is an open question whether LP can be solved in polynomial time in the real number model (cf. [43, p. 60]).

The feasibility problem $(\mathrm{F})$ belongs to NP in the real number model (since one can test in polynomial time whether a matrix is psd, for instance, using Gaussian elimination; in fact, for a rational matrix the running time is polynomial in its bitlength (cf. $[16$, p. 295])). However, it is not known whether problem (F) belongs to NP in the bit model. Indeed, in contrast with LP, it is not true that if a solution exists then one exists which is rational and has a polynomially bounded size. Consider, for instance, the following matrix:

$$
X:=\left(\begin{array}{cccc}
2 x & 2 & 0 & 0 \\
2 & x & 0 & 0 \\
0 & 0 & 2 & x \\
0 & 0 & x & 1
\end{array}\right)
$$

Then, $x=\sqrt{2}$ is the unique real for which $X \succeq 0$; hence, this is an instance where there is a real solution but no rational solution. Consider now the following matrix (taken from [35]):

$X=\left(\begin{array}{c|cc|c|cc|c|cc}x_{1}-2 & 0 & 0 & \ldots & 0 & 0 & \ldots & 0 & 0 \\ \hline 0 & 1 & x_{1} & \ldots & 0 & 0 & \ldots & 0 & 0 \\ 0 & x_{1} & x_{2} & \ldots & 0 & 0 & \ldots & 0 & 0 \\ \hline \vdots & \vdots & \vdots & \ddots & \vdots & \vdots & & \vdots & \vdots \\ \hline 0 & 0 & 0 & \ldots & 1 & x_{i} & \ldots & 0 & 0 \\ 0 & 0 & 0 & \ldots & x_{i} & x_{i+1} & \ldots & 0 & 0 \\ \hline \vdots & \vdots & \vdots & & \vdots & \vdots & \ddots & \vdots & \vdots \\ \hline 0 & 0 & 0 & \ldots & 0 & 0 & \ldots & 1 & x_{n-1} \\ 0 & 0 & 0 & \ldots & 0 & 0 & \ldots & x_{n-1} & x_{n}\end{array}\right)$.

Then, $X \succeq 0$ if and only if $x_{1} \geq 2$ and $x_{i+1} \geq x_{i}^{2}$ for $i=1, \ldots, n-1$; hence, $x_{n} \geq 2^{2^{n-1}}$ and thus any rational solution has exponential bitlength. More examples of "ill-conditioned" semidefinite problems can be found in [35].

However, Ramana [35] has developed an exact duality theory for SDP which enables him to show the following results: Problem (F) belongs to NP $\cap$ co-NP in the real number model. In the bit model, $(F)$ belongs to NP if and only if it belongs to co-NP; hence, $(\mathrm{F})$ is not NP-complete nor co-NP complete unless NP = co-NP.

Algorithms have been found that permit us to solve the optimization problem (1.5) approximatively in polynomial time; they are based on the ellipsoid method (cf. [16]) and interior-point methods (cf. [32], [3]). 
More precisely, set $K:=\left\{z \in \mathbf{R}^{m} \mid Q_{0}+\sum_{i=1}^{m} z_{i} Q_{i} \succeq 0\right\}$ and, given $\epsilon>0$, set $S(K, \epsilon):=\{y \mid \exists z \in K$ with $\|z-y\|<\epsilon\}$ ("the points that are in the $\epsilon$-neighborhood of $K$ ") and $S(K,-\epsilon):=\mathbf{R}^{m} \backslash S\left(\mathbf{R}^{m} \backslash K, \epsilon\right)$ ("the points that are at distance at least $\epsilon$ from the border of $\left.K^{\prime \prime}\right)$. Let $L$ denote the maximum bit size of the entries of the matrices $Q_{0}, \ldots, Q_{m}$. Assume that we know a constant $R>0$ such that either $K=\emptyset$ or $\exists z \in K$ with $\|z\| \leq R$. Then, the ellipsoid based algorithm, given rational $\epsilon>0$, either finds $y \in S(K, \epsilon)$ for which $c^{T} z \leq c^{T} y+\epsilon$ for all $z \in S(K,-\epsilon)$, or asserts that $S(K,-\epsilon)=\emptyset$. Its running time is polynomial in $n, m, L$, and $\log \epsilon$ and this algorithm is polynomial in the bit model.

Assume that we know a constant $R>0$ such that $\|z\| \leq R$ for all $z \in K$ and a point $z^{*} \in K$ for which $Q_{0}+\sum_{i=1}^{m} z_{i}^{*} Q_{i} \succ 0$ ( $z^{*}$ is "strictly feasible"). There is an interior-point algorithm which finds $y \in K$ strictly feasible such that $c^{T} y \geq$ $\max _{z \in K} c^{T} z-\epsilon$ in time polynomial in $n, m, L, \log \epsilon, \log R$, and in the bitlength of $z^{*}$. Note, however, that no polynomial bound has been established for the bitlengths of the intermediate numbers occurring in the algorithm.

Khachiyan and Porkolab have shown that problem $(\mathrm{F})$ and its analogue in rational numbers can be solved in polynomial time in the bit model for a fixed number $m$ of variables.

THEOREM 1.1

(i) [33] Problem (F) can be solved in polynomial time for any fixed $m$.

(ii) [24] The following problem can be solved in polynomial time for any fixed $m$ : Given $n \times n$ integral symmetric matrices $Q_{0}, Q_{1}, \ldots, Q_{m}$, find rational numbers $z_{1}, \ldots, z_{m}$ satisfying (1.4) or determine that no such numbers exist.

The result from Theorem 1.1 (ii) extends to the context of semidefinite programming the result of Lenstra [30] on the polynomial solvability of integer LP in fixed dimension.

1.3. Back to the matrix completion problem. Since the matrix completion problem is a special instance of SDP, it can be solved approximatively in polynomial time; specific interior-point algorithms for finding approximate psd and distance matrix completions have been developed, e.g., in [20], [11], [2], [31]. However, such algorithms are not guaranteed to find exact completions in polynomial time. This motivates our study in this paper of some classes of matrix completion problems that can be solved exactly in polynomial time.

As mentioned earlier, one of the difficulties in the complexity analysis of SDP arises from the fact that a rational SDP problem might have no rational solution. (Recall the example from (1.6).) This raises the following question in the context of matrix completion: If a rational partial matrix has a psd completion, does a rational completion always exist?

We do not know of a counterexample to this statement. On the other hand, we will show that the answer is positive, e.g., when the graph of specified entries is chordal or has minimum fill-in 1 (cf. Lemma 4.2). (Note that the answer is obviously positive if a pd completion exists.)

Motivated by the above discussion, let us define for each of the problems $(\mathrm{P})$ and (D) its rational analogue $\left(\mathrm{P}^{\mathrm{Q}}\right)$ and $\left(\mathrm{D}^{\mathrm{Q}}\right)$. Problem $\left(\mathrm{P}^{\mathrm{Q}}\right)$ is defined as follows:

Given a graph $G=(V, E)$, a subset $S \subseteq V$, and a rational vector $a \in \mathrm{Q}^{S \cup E}$, find a rational matrix $X$ satisfying (1.1) or determine that no such matrix exists.

When $S=V$ (i.e., all diagonal entries are specified), we denote the problem as $\left(\mathrm{P}_{s}^{Q}\right)$. Problem $\left(\mathrm{D}^{\mathrm{Q}}\right)$ is defined as follows: 
Given a graph $G=(V, E)$ and a rational vector $d \in \mathrm{Q}^{E}$, find a rational matrix $Y$ satisfying (1.3) or determine that no such matrix exists.

The complexity of the problems $(P),(D),\left(P^{Q}\right)$, and $\left(D^{Q}\right)$ is not known; in particular, it is not known whether they belong to NP in the bit model (they do trivially in the real number model). In this paper, we present some instances of graphs for which the completion problems can be solved in polynomial time. All our complexity results apply for the bit model (unless otherwise specified, as in section 5.3).

Recall that a graph is said to be chordal if it does not contain a circuit of length $\geq 4$ as an induced subgraph. Then, the minimum fill-in of graph $G$ is defined as the minimum number of edges needed to be added to $G$ in order to obtain a chordal graph. Note that computing the minimum fill-in of a graph is an NP-hard problem [44]. The following is the main result of sections 3 and 4 .

THEOREM 1.2. For any integer $m \geq 0$, problems $(\mathrm{P}),\left(\mathrm{P}^{\mathrm{Q}}\right),(\mathrm{D})$, and $\left(\mathrm{D}^{\mathrm{Q}}\right)$ can be solved in polynomial time (in the bit model) when restricted to the class of graphs whose minimum fill-in is equal to $m$.

The essential ingredients in the proof of Theorem 1.2 are the subcase $m=0$ (chordal case), Theorem 1.1, and the link (exposed in section 2.3) between psd matrices and distance matrices. In the chordal case, a simple combinatorial algorithm permits to solve the completion problem in polynomial time.

The psd matrix completion problem for chordal graphs has been extensively studied in the literature (cf. the survey of Johnson [19] for detailed references). In some sense, this problem has been solved by Grone et al. [15] who, building upon a result of Dym and Gohberg [12], have characterized when a vector $a$ indexed by the nodes and edges of a chordal graph admits a psd completion; cf. Theorem 3.1. From this follows the polynomial time solvability of problem $\left(\mathrm{P}_{s}\right)$ for chordal graphs. In fact, the result from Theorem 3.1 is proved in [15] in a constructive manner and, thus, yields an algorithm permitting to solve problem $\left(\mathrm{P}_{s}^{\mathrm{Q}}\right)$ for chordal graphs. This algorithm has a polynomial running time in the real number model; however, it has to be modified in order to achieve a polynomial running time in the bit model.

To summarize, the result from Theorem 1.2 also holds in the real number model for chordal graphs $(m=0)$; it would hold for all graphs having fixed minimum fill-in $m \geq 1$ if the result from Theorem 1.1 would remain valid in the real number model. ${ }^{1}$

We present in section 5.1 another class of graphs for which the matrix completion problem $\left(\mathrm{P}_{s}\right)$ can be solved in polynomial time (in the bit model). This class contains (generalized) circuits and wheels having a fixed length (and fatness); these graphs arise naturally when considering the polar approach to the psd matrix completion problem. Then, section 5.2 contains a brief description of this polar approach, together with some open questions and remarks. In the final section 5.3 , we consider the matrix completion problem for the class of graphs containing no homeomorph of $K_{4}$. (It contains circuits.) Then a condition characterizing existence of a psd or distance matrix completion exists which permits us to obtain a simple combinatorial algorithm solving the existence and construction problems in polynomial time in the real number model.

2. Preliminaries. We recall here some basic facts about Schur complements and Euclidean distance matrices that will be needed in the paper, and we make a few observations about psd completions.

\footnotetext{
${ }^{1}$ L. Porkolab [34] claims to have a proof of this fact.
} 
2.1. Schur complements. For a symmetric matrix $M$, set $\operatorname{In}(M):=(p, q, r)$, where $p$ (resp., $q, r$ ) denotes the number of positive (resp., negative, zero) eigenvalues of $M$. When $M \succeq 0$, a maximal nonsingular principal submatrix of $M$ is a nonsingular principal submatrix of $M$ of largest possible order, thus equal to the rank of $M$.

LEMMA 2.1. Let $M=\left(\begin{array}{cc}A & B \\ B^{T} & C\end{array}\right)$ be a symmetric matrix, where $A$ is nonsingular. Then,

$$
\operatorname{In}(M)=\operatorname{In}(A)+\operatorname{In}\left(C-B^{T} A^{-1} B\right)
$$

the matrix $C-B^{T} A^{-1} B$ is known as the Schur complement of $A$ in $M$. In particular, $M \succeq 0$ if and only if $A \succeq 0$ and $C-B^{T} A^{-1} B \succeq 0$. Moreover, if $M \succeq 0$ and if $A$ is a maximal nonsingular principal submatrix of $M$, then $C=B^{T} A^{-1} B$.

As a direct application, we have the following results which will be used at several occasions in the paper.

LEMMA 2.2. Let $X$ be a symmetric matrix having the block decomposition

$$
X=\frac{\ell}{m}\left(\begin{array}{ccc}
\ell & n & m \\
R & R^{T} & Z^{T} \\
Z & A & S \\
Z & S^{T} & D
\end{array}\right)
$$

where $T, R, Z, A, S, D$ are rational matrices of suitable orders; all entries of $X$ being specified except those of $Z$ that have to be determined in order to obtain $X \succeq 0$. Assume that

$$
X_{1}:=\left(\begin{array}{cc}
T & R^{T} \\
R & A
\end{array}\right) \succeq 0, X_{2}:=\left(\begin{array}{cc}
A & S \\
S^{T} & D
\end{array}\right) \succeq 0
$$

In the case when $n \geq 1$ and $A \neq 0$, let $A_{0}$ be a maximal nonsingular principal submatrix of $A$, and let

$$
A=\left(\begin{array}{cc}
A_{0} & B \\
B^{T} & C
\end{array}\right), X=\left(\begin{array}{cccc}
T & R_{0}^{T} & R_{1}^{T} & Z^{T} \\
R_{0} & A_{0} & B & S_{0} \\
R_{1} & B^{T} & C & S_{1} \\
Z & S_{0}^{T} & S_{1}^{T} & D
\end{array}\right)
$$

denote the corresponding block decompositions of $A$ and $X$. Then, $X \succeq 0$ if we set

$$
Z:=S_{0}^{T} A_{0}^{-1} R_{0}
$$

when $n \geq 1$ and $A \neq 0$, and $Z:=0$ otherwise.

Proof. The result follows using Lemma 2.1 after noting that the Schur complement of $A_{0}$ in $X$ is given by

$$
\begin{aligned}
& \left(\begin{array}{ccc}
T & R_{1}^{T} & Z^{T} \\
R_{1} & C & S_{1} \\
Z & S_{1}^{T} & D
\end{array}\right)-\left(\begin{array}{c}
R_{0}^{T} \\
B^{T} \\
S_{0}^{T}
\end{array}\right) A_{0}^{-1}\left(\begin{array}{ccc}
R_{0} & B & S_{0}
\end{array}\right) \\
= & \left(\begin{array}{ccc}
T-R_{0}^{T} A_{0}^{-1} R_{0} & R_{1}^{T}-R_{0}^{T} A_{0}^{-1} B & Z^{T}-R_{0}^{T} A_{0}^{-1} S_{0} \\
R_{1}-B^{T} A_{0}^{-1} R_{0} & C-B^{T} A_{0}^{-1} B & S_{1}-B^{T} A_{0}^{-1} S_{0} \\
Z-S_{0}^{T} A_{0}^{-1} R_{0} & S_{1}^{T}-S_{0}^{T} A_{0}^{-1} B & D-S_{0}^{T} A_{0}^{-1} S_{0}
\end{array}\right)
\end{aligned}
$$




$$
=\left(\begin{array}{ccc}
T-R_{0}^{T} A_{0}^{-1} R_{0} & 0 & Z^{T}-R_{0}^{T} A_{0}^{-1} S_{0} \\
0 & 0 & 0 \\
Z-S_{0}^{T} A_{0}^{-1} R_{0} & 0 & D-S_{0}^{T} A_{0}^{-1} S_{0}
\end{array}\right) .
$$

Indeed, the Schur complement $C-B^{T} A_{0}^{-1} B$ of $A_{0}$ in $A$ is equal to 0 since $A \succeq 0$ and $A_{0}$ is a maximal nonsingular principal submatrix of $A$; as $X_{1}, X_{2} \succeq 0$ this implies that $R_{1}-B^{T} A_{0}^{-1} R_{0}=S_{1}-B^{T} A_{0}^{-1} S_{0}=0$.

LeMma 2.3. Let $X$ be a symmetric matrix of the form

$$
X=\left(\begin{array}{cc}
T & R^{T} \\
R & A
\end{array}\right),
$$

where $A \succeq 0$ and $T$ is a symmetric matrix of order $\ell$ whose diagonal entries are all equal to some scalar $N$. Let $A_{0}$ be a maximal nonsingular principal submatrix of $A$ and let

$$
A=\left(\begin{array}{cc}
A_{0} & B \\
B^{T} & B^{T} A_{0}^{-1} B
\end{array}\right), X=\left(\begin{array}{ccc}
T & R_{0}^{T} & R_{1}^{T} \\
R_{0} & A_{0} & B \\
R_{1} & B^{T} & B^{T} A_{0}^{-1} B
\end{array}\right)
$$

denote the corresponding block decompositions of $A$ and $X$. Then, $X \succeq 0$ if and only if (i) $R_{1}=B^{T} A_{0}^{-1} R_{0}$ and (ii) $T-R_{0}^{T} A_{0}^{-1} R_{0} \succeq 0$. In particular, $X$ is pd if and only if $A$ and $T-R^{T} A^{-1} R$ are pd. Moreover, $T-R_{0}^{T} A_{0}^{-1} R_{0}$ is psd for $N$ large enough (namely, for $N$ greater or equal to the largest eigenvalue of $R_{0}^{T} A_{0}^{-1} R_{0}-T_{0}$, where $T_{0}$ has zero diagonal entries and as off-diagonal entries those of $T$ ).

2.2. Some observations about psd completions. Given a graph $G=(V, E)$, a subset $S \subseteq V$, a vector $a \in \mathrm{Q}^{S \cup E}$, and a scalar $N>0$, let $a^{N} \in \mathrm{Q}^{V \cup E}$ denote the extension of $a$ obtained by setting $a_{i}:=N$ for all $i \in V \backslash S$.

LEMMA 2.4. a is completable to a psd matrix if and only if $a^{N}$ is completable to a psd matrix for some $N>0$ (and then for all $N^{\prime} \geq N$ ).

Therefore, if one can "guess" a value $N$ to assign to the unspecified diagonal entries, then one can reduce the problem to the case when all diagonal entries are specified. This can be done when the graph $G$ of specified off-diagonal entries is chordal as we see later or if we look for a pd completion as the next result shows.

Lemma 2.5. Given $a \in \mathrm{Q}^{S \cup E}$, let $b:=\left(a_{i}(i \in S), a_{i j}(i j \in E, i, j \in S)\right)$ denote its restriction to the subgraph induced by $S$. Then, a has a pd completion if and only if $b$ has a pd completion.

Proof. Apply Lemma 2.3.

This result does not extend to psd completions (which contradicts a claim from [15] (psd case in Proposition 1)). Indeed, the partial matrix

$$
\left(\begin{array}{ccc}
? & 1 & -1 \\
1 & 1 & 1 \\
-1 & 1 & 1
\end{array}\right)
$$

has no psd completion while its lower principal $2 \times 2$ submatrix is psd.

A final observation is that if a partial matrix contains a fully specified row, then the completion problem can be reduced to considering a matrix of smaller order. Indeed, suppose that $A=\left(a_{i j}\right)$ is a partial symmetric matrix whose first row is fully specified. If $a_{11}<0$, then $A$ is not completable. If $a_{11}=0$, then $A$ is completable if and only if its first row is identically zero and its lower principal submatrix of order $n-1$ is completable. If $a_{11}>0$ then one can reduce to a problem of order $n-1$ by considering the Schur complement of $a_{11}$ in $A$. 
2.3. Euclidean distance matrices. The following connection (2.4) between psd and distance matrices has been established by Schoenberg [37]. Let $Y=\left(y_{i j}\right)_{i, j \in V}$ be a square symmetric matrix with zeros on its main diagonal and whose rows and columns are indexed by a set $V$, and let $i_{0}$ be a given element of $V$. Then, $\varphi_{i_{0}}(Y)$ denotes the square symmetric matrix $X=\left(x_{i j}\right)_{i, j \in V \backslash\left\{i_{0}\right\}}$ whose rows and columns are indexed by set $V \backslash\left\{i_{0}\right\}$ and whose entries are given by

$$
x_{i j}=\frac{1}{2}\left(y_{i_{0} i}+y_{i_{0} j}-y_{i j}\right) \text { for } i, j \in V \backslash\left\{i_{0}\right\} \text {. }
$$

Then,

$$
Y \text { is a distance matrix } \Longleftrightarrow \varphi_{i_{0}}(Y) \succeq 0 \text {. }
$$

(Indeed, a set of vectors $u_{i}(i \in V)$ forms a realization of the matrix $Y$ if and only if $\varphi_{i_{0}}(Y)$ is the Gram matrix of the vectors $u_{i}-u_{i_{0}}\left(i \in V \backslash\left\{i_{0}\right\}\right)$, which means that its $(i, j)$ th entry is equal to $\left(u_{i}-u_{i_{0}}\right)^{T}\left(u_{j}-u_{i_{0}}\right)$.) Thus, $\varphi_{i_{0}}$ establishes a linear bijection between the set of distance matrices of order $|V|$ and the set of psd matrices of order $|V|-1$. Relation (2.4) has a direct consequence for the corresponding matrix completion problems. Let $G=(V, E)$ be a graph and assume that $i_{0} \in V$ is a universal node, i.e., that $i_{0}$ is adjacent to all other nodes of $G$. Then, an algorithm permitting to solve the psd matrix completion problem for graph $G \backslash i_{0}$ can be used for solving the distance matrix completion problem for graph $G$ and vice versa. Indeed,

$$
\begin{aligned}
& Y \text { is a distance matrix completion of } d \in \mathbf{R}^{E} \\
& \Longleftrightarrow \varphi_{i_{0}}(Y) \text { is a psd completion of } \varphi_{i_{0}}(d) .
\end{aligned}
$$

(For the definition of $\varphi_{i_{0}}(d)$, use (2.3) restricted to the pairs ij with $i, j \in V \backslash\left\{i_{0}\right\}$, $i=j$, or $i \neq j$ with $i j$ edge of $G$.) For more information about connections between the two problems, see [21], [27].

3. The matrix completion problem for chordal graphs. We consider here the matrix completion problems for chordal graphs. First, we recall results from [15] and [4] yielding a good characterization for the existence of a completion; then, we see how they can be used for constructing a completion in polynomial time.

3.1. Characterizing existence of a completion. Let $G=(V, E)$ be a graph and let $a \in \mathrm{Q}^{V \cup E}$ be a vector; in the distance matrix case, the entries of $a$ indexed by $V$ (corresponding to the diagonal entries of a matrix completion) are assumed to be equal to zero. If $K \subseteq V$ is a clique in $G$ (i.e., any two distinct nodes in $K$ are joined by an edge in $G$ ), the entries $a_{i j}$ of vector $a$ are well-defined for all nodes $i, j \in K$; then, we let $a(K)$ denote the $|K| \times|K|$ symmetric matrix whose rows and columns are indexed by $K$ and with $i j$ th entry $a_{i j}$ for $i, j \in K$. Obviously, if $a$ is completable to a psd matrix, then $a$ satisfies

$$
a(K) \succeq 0 \text { for every maximal clique } K \text { in } G .
$$

Similarly, if $a$ is completable to a distance matrix, then $a$ satisfies

$$
a(K) \text { is a distance matrix for every maximal clique } K \text { in } G \text {. }
$$

The conditions (3.1) and (3.2) are not sufficient in general for ensuring the existence of a completion. For instance, if $G=(V, E)$ is a circuit and $a \in \mathrm{Q}^{V \cup E}$ has all its 
entries equal to 1 except one entry on an edge equal to -1 , then $a$ satisfies (3.1) but $a$ is not completable to a psd matrix. However, if $G$ is a chordal graph, then (3.1) and (3.2) suffice for ensuring the existence of a completion.

THEOREM 3.1. Let $G=(V, E)$ be a chordal graph and let $a \in \mathbf{R}^{V \cup E}$. If a satisfies (3.1), then a is completable to a psd matrix [15]; if a satisfies (3.2), then a is completable to a distance matrix [4]; moreover, if a is rational valued, then a admits a rational completion.

As the maximal cliques in a chordal graph can be enumerated in polynomial time [39] (cf. below) and as one can check positive semidefiniteness of a rational matrix in polynomial time (cf. [16, p. 295]), one can verify whether (3.1) holds in polynomial time when $G$ is chordal; in view of (2.4), one can also verify whether (3.2) holds in polynomial time when $G$ is chordal. This implies the next theorem.

Theorem 3.2. Problems $\left(\mathrm{P}_{s}\right)$ and $(\mathrm{D})$ can be solved in polynomial time for chordal graphs.

The proof given in [15], [4] for Theorem 3.1 is constructive; thus, it provides an algorithm for constructing a completion and, as we see below, a variant of it can be shown to have a polynomial running time. The proof is based on the following properties of chordal graphs. Let $G=(V, E)$ be a graph.

Then, $G$ is chordal if and only if it has a perfect elimination ordering; moreover, such an ordering can be found in polynomial time [36]. An ordering $v_{1}, \ldots, v_{n}$ of the nodes of a graph $G=(V, E)$ is called a perfect elimination ordering if, for every $j=1, \ldots, n-1$, the set of nodes $v_{k}$ with $k>j$ that are adjacent to $v_{j}$ induces a clique in $G$. For $j=1, \ldots, n-1$, let $K_{j}$ denote the clique consisting of node $v_{j}$ together with the nodes $v_{k}(k>j)$ that are adjacent to $v_{j}$; then the cliques $K_{1}, \ldots, K_{n-1}$ comprise all maximal cliques of a chordal graph $G$.

If $G$ is chordal and not a clique, then one can find (in polynomial time) an edge $e \notin E$ for which the graph $H:=G+e$ (obtained by adding $e$ to $G$ ) is chordal. (Indeed, let $i$ be the largest index in $[1, n]$ for which there exists $j>i$ such that $v_{i}$ and $v_{j}$ are not adjacent in $G$; then we can choose for $e$ the pair $i j$ as $v_{1}, \ldots, v_{n}$ remains a perfect elimination ordering for $H$.)

If $G$ is chordal then, for any $e \notin E$, there exists a unique maximal clique in $G+e$ containing edge $e[15]$ (easy to check).

Therefore, if $G$ is complete and not a clique, we can order the missing edges in $G$ as $e_{1}, \ldots, e_{p}$ in such a way that the graph $G_{q}:=\left(V, E \cup\left\{e_{1}, \ldots, e_{q}\right\}\right)$ is chordal for every $q=1, \ldots, p$. For $q=1, \ldots, p$, let $K_{q}$ be the unique maximal clique in $G_{q}$ containing edge $e_{q}$. Given $a \in \mathrm{Q}^{V \cup E}$ satisfying (3.1), set $G_{0}:=G$ and $x_{0}:=a$. We execute the following step for $q=1, \ldots, p$.

Find $z_{q} \in \mathbf{Q}$ for which the vector $x_{q}:=\left(x_{q-1}, z_{q}\right)$ of $\mathbf{Q}^{V \cup E\left(G_{q}\right)}$ satisfies

$$
x_{q}\left(K_{q}\right) \succeq 0 \text {. }
$$

This can be done in view of Lemma 2.2 (case $\ell=m=1$ ) applied to the matrix $X:=x_{q}\left(K_{q}\right)$ and one can choose for $z_{q}$ the rational value given by (2.2). Then, the final vector $x_{p}=\left(a, z_{1}, \ldots, z_{p}\right)$ provides a rational psd completion of $a$. This shows Theorem 3.1 in the psd case (the Euclidean distance matrix case being similar).

As mentioned earlier, the preprocessing step (find the suitable ordering $e_{1}, \ldots, e_{p}$ of the missing edges and the cliques $K_{q}$ ) can be done in polynomial time. Then, one can construct the values $z_{1}, \ldots, z_{p}$ yielding a psd completion of $a$ in $p \leq n^{2}$ steps. Therefore, the algorithm is polynomial in the real number model. In order to show polynomiality in the bit model, one has to verify that the encoding sizes 
of $z_{1}, \ldots, z_{p}$ remain polynomially bounded in terms of $n$ and the encoding size of $a$. This is, however, not clear. Indeed, both $R_{0}$ and $S_{0}$ in the definition of $z_{q}$ via (2.2) may involve some previously defined $z_{h}$ for $h<q$ (the same may hold for $A_{0}$ ); then, we have a quadratic dependence between $z_{q}$ and the previously defined $z_{1}, \ldots, z_{q-1}$ which may cause a problem when trying to prove that the encoding size of $z_{q}$ remains polynomially bounded. However, as we see below, the above algorithm can be modified to obtain a polynomial running time. The basic idea is that, instead of adding the missing edges one at a time, one adds them by "packets" consisting of edges sharing a common end node. Then, in view of Lemma 2.2 , one can specify simultaneously all the entries on these edges, which permits to achieve a linear dependency among the $z_{q}$ 's.

3.2. Constructing a psd completion in polynomial time. Let $G=(V, E)$ be a chordal graph and let $1, \ldots, n$ denote a perfect elimination ordering of its nodes. For $i \in[1, n]$, set

$$
J(i):=\{j \in[1, n]: j>i \text { and } i j \notin E\}
$$

and let $i_{1}>\cdots>i_{L}$ denote the elements $i \in[1, n]$ for which $J(i) \neq \emptyset$. For $\ell=$ $1, \ldots, L$, set $F_{\ell}:=\left\{i_{\ell} j \mid j \in J\left(i_{\ell}\right)\right\}$ and let $G_{\ell}$ denote the graph with node set $V$ and edge set $E \cup F_{1} \cup \cdots \cup F_{\ell}$. Hence, we have a sequence of graphs

$$
G_{0}:=G \subseteq G_{1} \subseteq \cdots \subseteq G_{\ell} \subseteq \cdots \subseteq G_{L}
$$

where each $G_{\ell}$ is chordal (since $1 \ldots n$ remains a perfect elimination ordering of its nodes) and $G_{L}$ is the complete graph. We now show that $G_{\ell}$ has only one maximal clique which is not a clique in $G_{\ell-1}$.

Lemma 3.3. For $\ell=1, \ldots, L$, there is a unique maximal clique $K_{\ell}$ in $G_{\ell}$ which is not a clique in $G_{\ell-1}$. Moreover, $J\left(i_{\ell}\right) \cup\left\{i_{\ell}\right\} \subseteq K_{\ell}$, the set $K_{\ell} \backslash\left\{i_{\ell}\right\}$ is a clique in $G_{\ell-1}$, and the set $K_{\ell} \backslash J\left(i_{\ell}\right)$ is a clique in $G$.

Proof. Let $K$ be a maximal clique in $G_{\ell}$ which is not a clique in $G_{\ell-1}$; then, $i_{\ell} \in K$ and $K \cap J\left(i_{\ell}\right) \neq \emptyset$; we first show that $J\left(i_{\ell}\right) \subseteq K$. For this, assume that $j, j^{\prime} \in J\left(i_{\ell}\right)$ with $j \in K$ and $j^{\prime} \notin K$. By maximality of $K$, there exists an element $i \in K$ such that $i$ and $j^{\prime}$ are not adjacent in $G_{\ell}$. Then, $i<i_{\ell}$ since the set $\left[i_{\ell}, n\right]$ is a clique in $G_{\ell}$. Therefore, the pairs $i j$ and $i i_{\ell}$ are edges of $G_{\ell}$ and, thus, of $G$. Since the ordering of the nodes is a perfect elimination ordering for $G$, this implies that $i_{\ell}$ and $j$ must be adjacent in $G$, yielding a contradiction.

Suppose now that $K, K^{\prime}$ are two distinct maximal cliques in $G_{\ell}$ such that $i_{\ell} \in$ $K \cap K^{\prime}$ and $J\left(i_{\ell}\right) \subseteq K \cap K^{\prime}$. Then, there exist nodes $i \in K \backslash K^{\prime}, i^{\prime} \in K^{\prime} \backslash K$ that are not adjacent in $G_{\ell}$. Given a node $j \in J\left(i_{\ell}\right)$, one can easily verify that $\left(i, i_{\ell}, i^{\prime}, j\right)$ is an induced circuit in $G_{\ell-1}$, which contradicts the fact that $G_{\ell-1}$ is chordal and, thus, shows unicity of the clique $K_{\ell}$. It is obvious that $K_{\ell} \backslash\left\{i_{\ell}\right\}$ is a clique in $G_{\ell-1}$. We now verify that $K_{\ell} \backslash J\left(i_{\ell}\right)$ is a clique in $G$. For this, note first that $i_{\ell}$ is adjacent to every node of $K_{\ell} \backslash\left(J\left(i_{\ell}\right) \cup\left\{i_{\ell}\right\}\right)$ in $G_{\ell}$ and, thus, in $G$. Suppose now that $x \neq y$ are two nodes in $K_{\ell} \backslash\left(J\left(i_{\ell}\right) \cup\left\{i_{\ell}\right\}\right)$ that are not adjacent in $G$. Then, as $x y$ is an edge of $G_{\ell-1}$, we have $x=i_{h}, y \in J\left(i_{h}\right)$ for some $h \leq \ell-1$ and, thus, $i_{\ell}<x, y$. As $i_{\ell}$ is adjacent to both $x$ and $y$ in $G$ this implies that $x$ and $y$ must be adjacent in $G$, yielding a contradiction.

We now describe the modified algorithm. Let $G=(V, E)$ be a chordal graph and let $a \in \mathbf{Q}^{V \cup E}$ satisfying (3.1). Setting $x_{0}:=a$, we execute the following step for $\ell=1, \ldots, L$. 
Find $z_{\ell} \in \mathrm{Q}^{F_{\ell}}$ for which the vector $x_{\ell}:=\left(x_{\ell-1}, z_{\ell}\right) \in \mathrm{Q}^{V \cup E\left(G_{\ell}\right)}$ satisfies

$$
x_{\ell}\left(K_{\ell}\right) \succeq 0 .
$$

Then, the final vector $x_{L}=\left(a, z_{1}, \ldots, z_{L}\right)$ provides a rational psd completion of $a$. For instance, we can choose for $z_{\ell}$ the value given by relation (2.2), applying Lemma 2.2 to the matrix $X:=x_{\ell}\left(K_{\ell}\right)$. (Indeed, in view of Lemma 3.3, $X_{1}=a\left(K_{\ell} \backslash J\left(i_{\ell}\right)\right) \succeq 0$ and $X_{2}=x_{\ell-1}\left(K_{\ell} \backslash\left\{i_{\ell}\right\}\right)$; thus, $X_{2} \succeq 0$ can be verified by induction.)

We verify that the encoding sizes of $z_{1}, \ldots, z_{L}$ are polynomially bounded in terms of $n$ and the encoding size of $a$. For this, we note that $z_{1}, \ldots, z_{L}$ are determined by a recurrence of the form

$$
z_{\ell}=S_{\ell}^{T} A_{\ell}^{-1} R_{\ell} \text { for } \ell=1, \ldots, L,
$$

where $R_{\ell}, A_{\ell}, S_{\ell}$ are matrices of (appropriate) orders $\leq n$. A crucial observation is that all entries of $R_{\ell}$ and $A_{\ell}$ belong to the set, denoted as $\mathcal{A}$, of entries of $a$ (as $K_{\ell} \backslash J\left(i_{\ell}\right)$ is a clique in $G$, by Lemma 3.3), while the entries of $S_{\ell}$ belong to the set $\mathcal{A} \cup \mathcal{Z}_{\ell-1}$, where $\mathcal{Z}_{\ell-1}$ denotes the set of entries of $\left(z_{1}, \ldots, z_{\ell-1}\right)$.

For $r \in \mathrm{Q}$, let $\langle r\rangle$ denote the encoding size of $r$, i.e., the number of bits needed to encode $r$ in binary notation and, for a vector $x=\left(x_{1}, \ldots, x_{p}\right) \in \mathrm{Q}^{p}$, set $s(x):=$ $\max \left(\left\langle x_{1}\right\rangle, \ldots,\left\langle x_{p}\right\rangle\right)$. One can verify that, for two vectors $x, y \in \mathrm{Q}^{p},\left\langle x^{T} y\right\rangle \leq\langle n\rangle+$ $s(x)+s(y)$. Let $S_{a}$ denote the maximum encoding length of the entries of vector $a$ and, for $\ell=1, \ldots, L$, set $S_{\ell}:=\max \left(\langle z\rangle \mid z \in \mathcal{Z}_{\ell}\right)$. We derive from (3.6) that

$$
S_{\ell} \leq\langle n\rangle+s\left(A_{\ell}^{-1} R_{\ell}\right)+S_{a}+S_{\ell-1}
$$

for all $\ell$ (setting $S_{0}:=0$ ). This implies that

$$
S_{L} \leq L\left(S_{a}+\langle n\rangle\right)+\sum_{\ell=1}^{L} s\left(A_{\ell}^{-1} R_{\ell}\right) .
$$

As $L \leq n$, we obtain that all encoding sizes of $z_{1}, \ldots, z_{L}$ are polynomially bounded in terms of $n$ and the encoding size of $a$. (We also use here the fact that the entries of $A_{\ell}^{-1}$ are polynomially bounded in the input size; cf. [16, Chapter 1.3].) Thus, we have shown the following theorem.

THEOREM 3.4. Problem $\left(\mathrm{P}_{s}^{\mathrm{Q}}\right)$ can be solved in polynomial time for chordal graphs.

We finally indicate how to solve the general problem when some diagonal entries are unspecified.

THEOREM 3.5. Problems $(\mathrm{P})$ and $\left(\mathrm{P}^{\mathrm{Q}}\right)$ can be solved in polynomial time for chordal graphs.

Proof. Let $G=(V, E)$ be a chordal graph, let $S \subseteq V$, and let $a \in \mathrm{Q}^{S \cup E}$ satisfying $a(K) \succeq 0$ for each maximal clique $K \subseteq S$. (Else, we can conclude that $a$ is not completable.) Following Lemma 2.4, we search for a scalar $N>0$ such that $a$ is completable if and only if its extension $a^{N} \in \mathrm{Q}^{V \cup E}$ (assigning value $N$ to the unspecified diagonal entries) is completable or, equivalently, $a^{N}(K) \succeq 0$ for all maximal cliques $K$ in $G$. Note that each matrix $a^{N}(K)$ has the same form as matrix $X$ from Lemma 2.3. Therefore, such $N$ exists if and only if the linear condition (i) from Lemma 2.3 holds for each clique $K$ and an explicit value for $N$ can be constructed as indicated in Lemma 2.3. Once $N$ has been determined, we proceed with completing $a^{N}$ by applying the algorithm presented above.

To conclude note that the algorithm presented in this section outputs a pd completion if one exists. 
3.3. Constructing a distance matrix completion. The distance matrix completion problem for chordal graphs can be solved in an analogous manner. Namely, let $G=(V, E)$ be a chordal graph, let

$$
G_{0}:=G \subseteq \cdots \subseteq G_{\ell} \subseteq \cdots \subseteq G_{L}
$$

be the sequence of chordal graphs from (3.4), let $K_{\ell}(\ell=1, \ldots, L)$ be the cliques constructed in Lemma 3.3, and let $a \in \mathrm{Q}^{E}$ satisfying (3.2). Setting $a_{0}:=a$, we execute the following step for $\ell=1, \ldots, L$ :

Find $z_{\ell} \in \mathrm{Q}^{F_{\ell}}$ for which the vector $x_{\ell}:=\left(a_{\ell-1}, z_{\ell}\right) \in \mathrm{Q}^{E\left(G_{\ell}\right)}$ satisfies

$$
x_{\ell}\left(K_{\ell}\right) \text { is a distance matrix. }
$$

Then, the final vector $x_{L}=\left(a, z_{1}, \ldots, z_{L}\right)$ provides a distance matrix completion of $a$. The above step can be performed as follows. If $K_{\ell}=J\left(i_{\ell}\right) \cup\left\{i_{\ell}\right\}$, then we let $z_{\ell}$ be defined by $z_{\ell}(j):=x_{\ell-1}\left(j_{0}, j\right)$ for $j \in J\left(i_{\ell}\right)$, where $j_{0}$ is a given element of $J\left(i_{\ell}\right)$. Otherwise, let $j_{0} \in K_{\ell} \backslash\left(J\left(i_{\ell}\right) \cup\left\{i_{\ell}\right\}\right)$; then $j_{0}$ is a universal node in $G\left[K_{\ell}\right]$, the subgraph of $G$ induced by $K_{\ell}$. Therefore, in view of relation (2.5), we can find $z_{\ell}$ satisfying (3.7) by applying Lemma 2.2. The polynomial running time of the above algorithm follows from the polynomial running time of the corresponding algorithm in the psd case. Thus, we have shown the following theorem.

THEOREM 3.6. Problem $\left(D^{Q}\right)$ can be solved in polynomial time for chordal graphs.

4. The matrix completion problem for graphs with fixed minimum fillin. In this section we describe an algorithm permitting us to solve problems $(\mathrm{P})$, $\left(\mathrm{P}^{\mathbf{Q}}\right),(\mathrm{D})$, and $\left(\mathrm{D}^{\mathbf{Q}}\right)$ in polynomial time for the graphs having minimum fill-in $m$, where $m \geq 1$ is a given integer. This algorithm is based on Theorems 1.1, 3.1, 3.2, 3.4 , and 3.6 .

Let $G=(V, E)$ be a graph with minimum fill-in $m$, let $S \subseteq V$ and let $a \in \mathrm{Q}^{S \cup E}$ be given. (Again we assume that $a_{i}=0$ for $i \in V$ in the distance matrix case.) We first execute the following step.

Step 0 . Find edges $e_{1}, \ldots, e_{m} \notin E$ for which the graph $H:=\left(V, E \cup\left\{e_{1}, \ldots, e_{m}\right\}\right)$ is chordal and find the maximal cliques $K_{1}, \ldots, K_{p}$ in $H$. (Such edges exist since $G$ has minimum fill-in $m$ and they can be found in polynomial time, simply by enumeration as $m$ is fixed. The maximal cliques in $H$ can also be enumerated in polynomial time since $H$ is chordal and, moreover, $p \leq n$.)

Then, we perform step $x$ in order to solve problem $(x)$ for $x=\mathrm{P}, \mathrm{P}^{\mathbf{Q}}, \mathrm{D}, \mathrm{D}^{\mathbf{Q}}$.

Step $P$. Determine whether there exist real numbers $z_{1}, \ldots, z_{m}, z_{m+1}$ for which the vector $x \in \mathrm{Q}^{V \cup E(H)}$ defined by $x_{i}:=a_{i}(i \in S), x_{i}:=z_{m+1}(i \in V \backslash S), x_{i j}=a_{i j}$ $(i j \in E)$, and $x_{e_{h}}:=z_{h}(h=1, \ldots, m)$ satisfies

$$
x\left(K_{1}\right) \succeq 0, \ldots, x\left(K_{p}\right) \succeq 0 .
$$

Step $D$. Determine whether there exist real numbers $z_{1}, \ldots, z_{m}$ for which the vector $x \in \mathrm{Q}^{E(H)}$ defined by $x_{i j}=a_{i j}(i j \in E)$, and $x_{e_{h}}:=z_{h}(h=1, \ldots, m)$ satisfies

$$
x\left(K_{1}\right), \ldots, x\left(K_{p}\right) \text { are distance matrices. }
$$

Then, $a$ has a completion if and only if the answer in Step P or D is positive.

Step $P^{\mathbf{Q}}$. Find rational numbers $z_{1}, \ldots, z_{m}, z_{m+1}$ for which (4.1) holds or determine that no such numbers exist; if they exist, find a rational psd completion of $x$. 
Step $D^{\mathbf{Q}}$. Find rational numbers $z_{1}, \ldots, z_{m}$ for which (4.2) holds or determine that no such numbers exist; if they exist, find a rational distance matrix completion of $x$.

Steps $\mathrm{P}$ and $\mathrm{P}^{\mathrm{Q}}$ can be executed in the following manner. Let $M$ denote the block diagonal matrix with the $p$ matrices $x\left(K_{1}\right), \ldots, x\left(K_{p}\right)$ as diagonal blocks (and zeros elsewhere). Hence, $M$ has order $\left|K_{1}\right|+\cdots+\left|K_{p}\right| \leq n^{2}$ and (4.1) holds if and only if $M \succeq 0$. Clearly, the matrix $M$ can be written under the form

$$
M=Q_{0}+z_{1} Q_{1}+\cdots+z_{m+1} Q_{m+1},
$$

where $Q_{1}, \ldots, Q_{m+1}$ are symmetric matrices with $(0,1)$-entries and $Q_{0}$ is a symmetric matrix whose nonzero entries belong to the set of entries of $a$. Therefore, in view of Theorem 1.1, one can determine the existence of $z_{1}, \ldots, z_{m+1}$ satisfying (4.1) in polynomial time. Then, finding a rational psd completion of $x$ in Step $\mathrm{P}^{\mathbf{Q}}$ can be done in polynomial time in view of Theorem 3.4.

In the distance matrix case, we use the following construction for distance matrices. For $a=1, \ldots, p$, let $D_{a}$ be a square symmetric matrix whose rows and columns are indexed by set $V_{a}$ and let $i_{a}$ be a given element of $V_{a}$. We construct a new matrix $D$, denoted as $D_{1} \oplus \cdots \oplus D_{p}$, whose rows and columns are indexed by set $V_{1} \cup \cdots \cup V_{p}$ and whose entries are given by

$$
D(i, j)= \begin{cases}D_{a}(i, j) & \text { if } i, j \in V_{a}, a \in[1, p], \\ D_{a}\left(i, i_{a}\right)+D_{b}\left(j, i_{b}\right) & \text { if } i \in V_{a}, j \in V_{b}, a \neq b \in[1, p] .\end{cases}
$$

Lemma 4.1. $D_{1} \oplus \cdots \oplus D_{p}$ is a distance matrix if and only if $D_{1}, \ldots, D_{p}$ are distance matrices.

Proof. The "only if" part is obvious. Conversely, assume that $D_{1}, \ldots, D_{p}$ are distance matrices; we show that $D:=D_{1} \oplus \cdots \oplus D_{p}$ is a distance matrix. For $a \in[1, p]$, let $u_{i}^{a} \in \mathbf{R}^{n_{a}}\left(i \in V_{a}\right)$ be vectors providing a realization of $D_{a}$; we can assume without loss of generality that $u_{i_{a}}^{a}=0$. Then, we construct a sequence of vectors $w_{i} \in \mathbf{R}^{n_{1}+\cdots+n_{p}}\left(i \in \bigcup_{a=1}^{p} V_{a}\right)$ by setting $w_{i}:=\left(0_{n_{1}}, \ldots, 0_{n_{a-1}}, u_{i}^{a}, 0_{n_{a+1}}, \ldots, 0_{n_{p}}\right)$ for $i \in V_{a} .\left(0_{n}\right.$ denotes the zero vector in $\mathbf{R}^{n}$.) One can easily verify that the vectors $w_{i}$ provide a realization of $D$.

Steps $\mathrm{D}$ and $\mathrm{D}^{\mathbf{Q}}$ can be performed as follows. Let $M:=x\left(K_{1}\right) \oplus \cdots \oplus x\left(K_{p}\right)$ denote the matrix indexed by $K_{1} \cup \cdots \cup K_{p}$ constructed as indicated in relation (4.3). Clearly, $M$ can be written under the form

$$
M=Q_{0}+z_{1} Q_{1}+\cdots+z_{m} Q_{m},
$$

where $Q_{1}, \ldots, Q_{m}$ are symmetric matrices with entries in $\{0,1\}$ and $Q_{0}$ is a symmetric matrix whose nonzero entries are sums of at most two entries of $a$. Let $i_{0}$ be a given element of $K_{1} \cup \cdots \cup K_{p}$. Then,

$$
\varphi_{i_{0}}(M)=\varphi_{i_{0}}\left(Q_{0}\right)+z_{1} \varphi_{i_{0}}\left(Q_{1}\right)+\cdots+z_{m} \varphi_{i_{0}}\left(Q_{m}\right) .
$$

Hence, (4.2) holds if and only if matrix $M$ is a distance matrix (by Lemma 4.1) or, equivalently, if and only if $\varphi_{i_{0}}(M)$ is positive semidefinite (by relation (2.4)). Therefore, in view of Theorems 3.2 and 3.6 , Steps $D$ and $D^{Q}$ can be executed in polynomial time. This completes the proof of Theorem 1.2.

LEMMA 4.2. When the minimum fill-in $m$ is equal to 1 , existence of a completion implies existence of a rational one. 
Proof. To see it, suppose first that all diagonal entries are specified; then, Steps $\mathrm{P}$ and $\mathrm{P}^{\mathbf{Q}}$ can be executed in an elementary manner. Indeed, each matrix $x\left(K_{i}\right)$ $(i=1, \ldots, p)$ has at most one unspecified entry $z_{1}$. Hence, the set of scalars $z_{1}$ for which $x\left(K_{i}\right) \succeq 0$ is an interval of the form $I_{i}=\left[\beta_{i}-\sqrt{\alpha_{i}}, \beta_{i}+\sqrt{\alpha_{i}}\right]$ where $\alpha_{i}, \beta_{i} \in \mathbf{Q}$ (easy to see from Lemma 2.2). Therefore, (4.1) holds if and only if $z_{1} \in \bigcap_{i=1}^{p} I_{i}=[u, v]$, where $u:=\max _{i}\left(\beta_{i}-\sqrt{\alpha_{i}}\right)$ and $v:=\min _{i}\left(\beta_{i}+\sqrt{\alpha_{i}}\right)$. Moreover, if there is a completion (i.e., if $u \leq v$ ), then one can find one with $z_{1}$ rational. This is obvious if $u<v$ and, if $u=v$, this follows from the fact (easy to verify) that

$$
\beta-\sqrt{\alpha}=\beta^{\prime}+\sqrt{\alpha^{\prime}}, \alpha, \alpha^{\prime}, \beta, \beta^{\prime} \in \mathrm{Q} \Longrightarrow \sqrt{\alpha}, \sqrt{\alpha^{\prime}} \in \mathrm{Q} .
$$

Suppose now some diagonal entries are unspecified. If there is a completion with value $z_{2}$ at the unspecified diagonal entries, then we can assume that $z_{2}$ is rational (replacing if necessary $z_{2}$ by a larger rational number). Then, by the above discussion, the off-diagonal unspecified entry $z_{1}$ can also be chosen to be rational.

5. Further results and open questions. We present in section 5.1 another class of graphs for which the completion problem can be solved in polynomial time (in the bit model). Then, we discuss in section 5.2 some open questions arising when considering a polar approach to the psd completion problem. Finally, we describe in section 5.3 a simple combinatorial algorithm permitting us to solve the completion problem in polynomial time (in the real number model) for the class of graphs containing no homeomorph of $K_{4}$.

5.1. Another class of polynomial instances. We present here another class of graphs for which the psd matrix completion problem $\left(\mathrm{P}_{s}\right)$ can be solved in polynomial time. Given two integers $p, q \geq 1$, let $\mathcal{G}_{p, q}$ be the class consisting of the graphs $G=(V, E)$ satisfying the following properties. There exist two disjoint subsets $V_{1}, V_{2}$ of $V$ such that $\min \left(\left|V_{1}\right|,\left|V_{2}\right|\right)=p$, the set $F:=\left\{i j \mid i \in V_{1}, j \in V_{2}\right\}$ is disjoint from $E$, the graph

$$
H:=(V, E \cup F)
$$

is chordal, and $H$ has $q$ maximal cliques that are not cliques in $G$.

THEOREM 5.1. Given integers $p, q \geq 1$, the psd completion problem $\left(\mathrm{P}_{s}\right)$ can be solved in polynomial time (in the bit model) over the class $\mathcal{G}_{p, q}$.

Examples of graphs belonging to class $\mathcal{G}_{p, q}$ arise from circuits, wheels, and some generalizations. A generalized circuit of length $n$ is defined in the following manner: its node set is $U_{1} \cup \cdots \cup U_{n}$ with two nodes $u \in U_{i}, v \in U_{j}$ being adjacent if and only if $i=j$ or $j=i+1$ (modulo $n$ ); a generalized wheel of length $n$ is obtained by adding a set $U_{0}$ (the center of the wheel) of pairwise adjacent nodes to a generalized circuit of length $n$ and making each node in $U_{0}$ adjacent to each node in $U_{1} \cup \cdots \cup U_{n}$. Call a generalized circuit or wheel $p$-fat if $\min \left(\left|U_{i}\right|: i=1, \ldots, n\right)=p$. Cf. Figure 5.1 for an example. Then, any $p$-fat generalized circuit or wheel of length $q+2$ belongs to $\mathcal{G}_{p, q}$. We will see in section 5.2 that generalized circuits and wheels arise as basic objects when studying the matrix completion problem on graphs of small order.

The proof of Theorem 5.1 is based on the following result of Barvinok [8], which shows that one can test feasibility of a system of quadratic equations in polynomial time for any fixed number of equations. ${ }^{2}$

\footnotetext{
${ }^{2}$ In [8] Barvinok considers the homogeneous case, where each equation is of the form $f_{i}(x)=$ $x^{T} A_{i} x=0$ for some symmetric matrix $A_{i}$. However, the general nonhomogeneous case can be derived from it [9].
} 


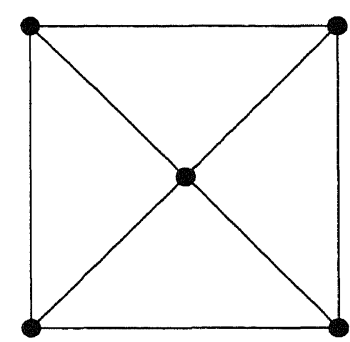

(a)

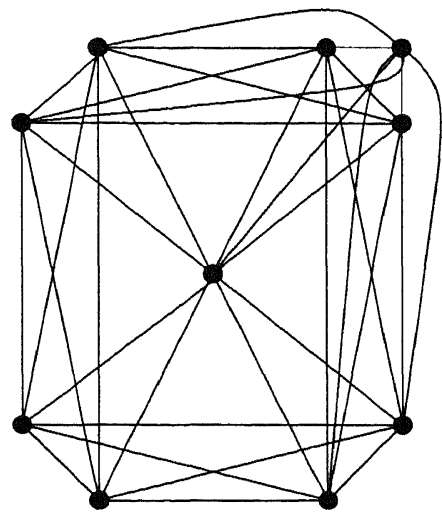

(b)

FIG. 5.1. (a) The wheel of length 4; (b) a 2-fat generalized wheel of length 4.

Theorem 5.2. For $i=1, \ldots, m$, let $f_{i}(x)=x^{T} A_{i} x+b_{i}^{T} x+c_{i}$ be a quadratic polynomial in $x \in \mathbf{R}^{n}$, where $A_{i}$ is an $n \times n$ symmetric matrix, $b_{i} \in \mathbf{R}^{n}$, and $c_{i} \in \mathbf{R}$. One can test feasibility of the system $f_{i}(x)=0$ for $i=1, \ldots, m$ in polynomial time (in the bit model) for any given $m$.

Proof of Theorem 5.1. Let $G=(V, E)$ be a graph in class $\mathcal{G}_{p, q}$ and let $a \in \mathbf{R}^{V \cup E}$ be given. We are also given the sets $V_{1}$ and $V_{2}$ for which, say, $p=\left|V_{1}\right| \leq\left|V_{2}\right|$ and adding to $G$ all edges in $F:=\left\{i j \mid i \in V_{1}, j \in V_{2}\right\}$ creates a chordal graph $H$. We show that deciding whether $a$ can be completed to a psd matrix amounts to testing the feasibility of a system of $m$ quadratic polynomials where $m$ depends only on $p$ and $q$. As $H$ is chordal, $a$ is completable to a psd matrix if and only if there exists a matrix $Z$ of order $V_{2} \times V_{1}$ for which $x:=(a, Z) \in \mathbf{R}^{V \cup E \cup F}$ satisfies $x(K) \succeq 0$ for each maximal clique $K$ in $H$. We assume that $x(K)=a(K) \succeq 0$ for each maximal clique $K$ of $H$ contained in $G$. (Else, we can conclude that $a$ is not completable.) Consider now a maximal clique $K$ of $H$ which is not contained in $G$. Then, $x(K)$ has the form

$$
x(K)=\begin{aligned}
& V_{1} \cap K \\
& V_{0} \cap K \\
& V_{2} \cap K
\end{aligned}\left(\begin{array}{ccc}
T & V_{0} \cap K & V_{2} \cap K \\
R & R^{T} & Z_{K}^{T} \\
Z_{K} & S^{T} & S
\end{array}\right)
$$

setting $V_{0}:=V \backslash\left(V_{1} \cup V_{2}\right)$ and $Z_{K}:=Z\left[V_{2} \cap K, V_{1} \cap K\right]$, the submatrix of $Z$ with row indices in $V_{2} \cap K$ and column indices in $V_{1} \cap K$. With the notation of Lemma 2.2 , we obtain that $x(K) \succeq 0$ if and only if the following matrix

$$
M_{K}:=\left(\begin{array}{cc}
T-R_{0}^{T} A_{0}^{-1} R_{0} & Z_{K}^{T}-R_{0}^{T} A_{0}^{-1} S_{0} \\
Z_{K}-S_{0}^{T} A_{0}^{-1} R_{0} & D-S_{0} A_{0}^{-1} S_{0}
\end{array}\right)
$$

is psd. (We have assumed that $A \succeq 0$.) We can apply again a Schur decomposition to matrix $M_{K}$ in order to reformulate the condition on $Z$. Setting $T_{K}:=T-R_{0}^{T} A_{0}^{-1} R_{0}$, $Z^{\prime}:=Z_{K}-S_{0}^{T} A_{0}^{-1} R_{0}$, and $D^{\prime}:=D-S_{0} A_{0}^{-1} S_{0}$, we have that $M_{K}=\left(\begin{array}{cc}T_{K} & Z^{\prime T} \\ Z^{\prime} & D^{\prime}\end{array}\right)$. Let 
$D_{0}^{\prime}$ be a largest nonsingular submatrix of $D^{\prime}$ and let

$$
D^{\prime}=\left(\begin{array}{cc}
D_{0}^{\prime} & E \\
E^{T} & F
\end{array}\right), M_{K}=\left(\begin{array}{ccc}
T_{K} & Z_{0}^{\prime T} & Z_{1}^{\prime T} \\
Z_{0}^{\prime} & D_{0}^{\prime} & E \\
Z_{1}^{\prime} & E^{T} & F
\end{array}\right)
$$

denote the corresponding block decompositions of $D^{\prime}$ and $M_{K}$. Taking the Schur complement of $D_{0}^{\prime}$ in $M_{K}$, we obtain that $M_{K} \succeq 0$ if and only if

$$
D^{\prime} \succeq 0, T_{K}-Z_{0}^{\prime T} D_{0}^{\prime-1} Z_{0}^{\prime} \succeq 0, \text { and } Z_{1}^{\prime}-E^{T} D_{0}^{\prime-1} Z_{0}^{\prime}=0 .
$$

Let $Y_{K}:=Z\left[V_{2}, V_{1} \cap K\right]$ denote the column submatrix of $Z$ with column indices in $V_{1} \cap K$ and set

$$
V_{K}:=\begin{gathered}
V_{1} \cap K \\
V_{2} \backslash K
\end{gathered}\left(\begin{array}{c}
S_{0}^{T} A_{0}^{-1} R_{0} \\
0
\end{array}\right), Q_{K}:=\left(\begin{array}{cc}
D_{0}^{-1} & 0 \\
0 & 0
\end{array}\right), G_{K}:=\left(\begin{array}{lll}
-E^{T} D_{0}^{\prime-1} & I & 0
\end{array}\right) .
$$

Then,

$$
\begin{gathered}
T_{K}-Z_{0}^{\prime T} D_{0}^{\prime-1} Z_{0}^{\prime}=T_{K}-\left(Y_{K}-V_{K}\right)^{T} Q_{K}\left(Y_{K}-V_{K}\right), \\
Z_{1}^{\prime}-E^{T} D_{0}^{\prime-1} Z_{0}^{\prime}=G_{K}\left(Y_{K}-V_{K}\right) .
\end{gathered}
$$

Therefore, the condition $x(K) \succeq 0$ can be rewritten as the system

$$
\left\{\begin{array}{l}
(1 \mathrm{~K}) T_{K}-\left(Y_{K}-V_{K}\right)^{T} Q_{K}\left(Y_{K}-V_{K}\right) \succeq 0, \\
(2 \mathrm{~K}) G_{K}\left(Y_{K}-V_{K}\right)=0
\end{array}\right.
$$

where $T_{K}, V_{K}, Q_{K}$ are matrices depending on input data $a$. We can reformulate condition $(1 \mathrm{~K})$ as an equation by introducing a new square matrix $S_{K}$ of order $V_{1} \cap K$ as "slack variable"; namely, rewrite $(1 \mathrm{~K})$ as

$$
\left(1^{\prime} \mathrm{K}\right) T_{K}-\left(Y_{K}-V_{K}\right)^{T} Q_{K}\left(Y_{K}-V_{K}\right)-S_{K}^{T} S_{K}=0 .
$$

Now, let $z_{1}, \ldots, z_{p} \in \mathbf{R}^{V_{2}}$ denote the columns of matrix $Z$, and let $s_{i}^{K}$ (for $i \in V_{1} \cap K$ ) denote the columns of matrix $S_{K}$ for each clique $K$. Then, condition $\left(1^{\prime} \mathrm{K}\right)$ can be expressed as a system of $\left(\begin{array}{c}\left|V_{1} \cap K\right|+1 \\ 2\end{array}\right)$ equations of the form

$$
f\left(z_{1}, \ldots, z_{p}, s_{i}^{K}\left(i \in V_{1} \cap K\right)\right)=0,
$$

where $f$ is a quadratic polynomial, similarly for condition $(2 \mathrm{~K})$. The total number of quadratic equations obtained in this manner depends only on $p$ and $q$. Therefore, in view of Theorem 5.2, one can check feasibility of this system in polynomial time when $p$ and $q$ are fixed.

Let $\mathcal{G}_{p, q}^{\prime}$ denote the subclass of $\mathcal{G}_{p, q}$ consisting of the graphs $G$ for which every maximal clique of $H$ (the chordal extension of $G$ ) which is not a clique of $G$ is not contained in $V_{1} \cup V_{2}$. Then, the Euclidean distance matrix completion problem can be solved in polynomial time over the class $\mathcal{G}_{p, q}^{\prime}$ for any fixed $p$ and $q$. The proof is similar to that of Theorem 5.1, since we can get back to the psd case using relation (2.4) (a matrix and its image under $\varphi_{i_{0}}$ having the same pattern of unknown entries if $i_{0}$ belongs to $\left.V \backslash\left(V_{1} \cup V_{2}\right)\right)$. In particular, the Euclidean distance matrix completion 


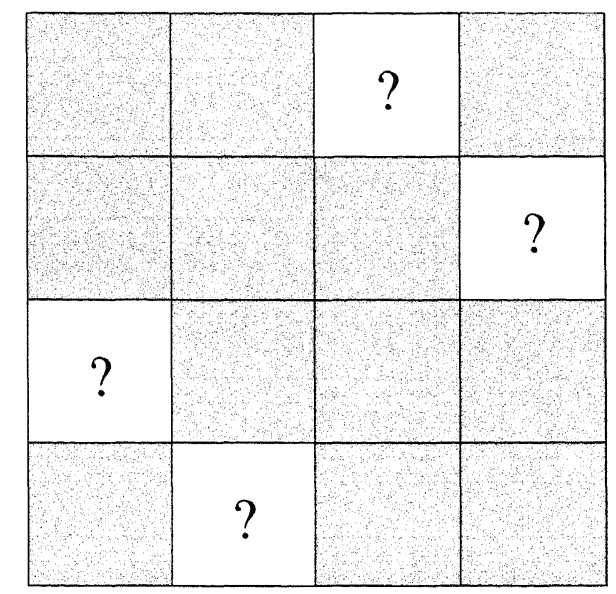

FIG. 5.2. The matrix completion problem for generalized circuits of length 4 .

problem can be solved in polynomial time for generalized circuits of length 4 and fixed fatness, or for generalized wheels (with a nonempty center) of fixed length and fatness.

The complexity of the psd completion problem for generalized wheels and circuits is not known; in fact, in view of the remark made at the end of section 2.2 , it suffices to consider circuits. In view of Theorem 5.1, the problem is polynomial if we fix the length and the fatness of the circuit. It would be particularly interesting to determine the complexity of the completion problem for generalized circuits of length 4 and unrestricted fatness. This problem can be reformulated as follows: Determine whether and how one can fill the unspecified entries in the blocks marked "?" of the matrix $X$ shown in Figure 5.2, so as to obtain $X \succeq 0$. (All entries are assumed to be specified in the grey blocks.) Indeed, as will be seen in section 5.2, these graphs constitute in some sense the next case to consider after chordal graphs.

5.2. A polar approach to the completion problem. Given a graph $G=$ $(V, E)$, consider the cone $C_{G}$ consisting of the matrices $X=\left(x_{i j}\right)_{i, j \in V}$ satisfying $X \succeq 0$ and $x_{i j}=0$ for all $i \neq j$ such that $i j \notin E$. Call $X \in C_{G}$ extremal if $X$ lies on an extremal ray of the cone $C_{G}$ (i.e., $X=Y+Z$ with $Y, Z \in C_{G}$ implies that $Y=\alpha X$ for some $\alpha \geq 0$ ) and define the order of $G$ as the maximum rank of an extremal matrix $X \in \bar{C}_{G}$. It is shown in [1] that $a \in \mathbf{R}^{V \cup E}$ is completable to a psd matrix if and only if $a$ satisfies

$$
\sum_{i j \in E} a_{i j} x_{i j}+\sum_{i \in V} a_{i} x_{i i} \geq 0
$$

for every extremal matrix $X=\left(x_{i j}\right) \in C_{G}$. One might suspect that the psd matrix completion problem is somewhat easier to solve for graphs having a small order since the extremal matrices in $C_{G}$ have then a small rank. Indeed, the graphs of order 1 are precisely the chordal graphs for which the problem is polynomially solvable. On the other hand, a circuit of length $n$ has order $n-2$ which is the highest possible order for a graph on $n$ nodes. Moreover, if $i_{0}$ is a universal node in a graph $G$, then both graphs $G$ and $G \backslash i_{0}$ have the same order, which corroborates the observation made 


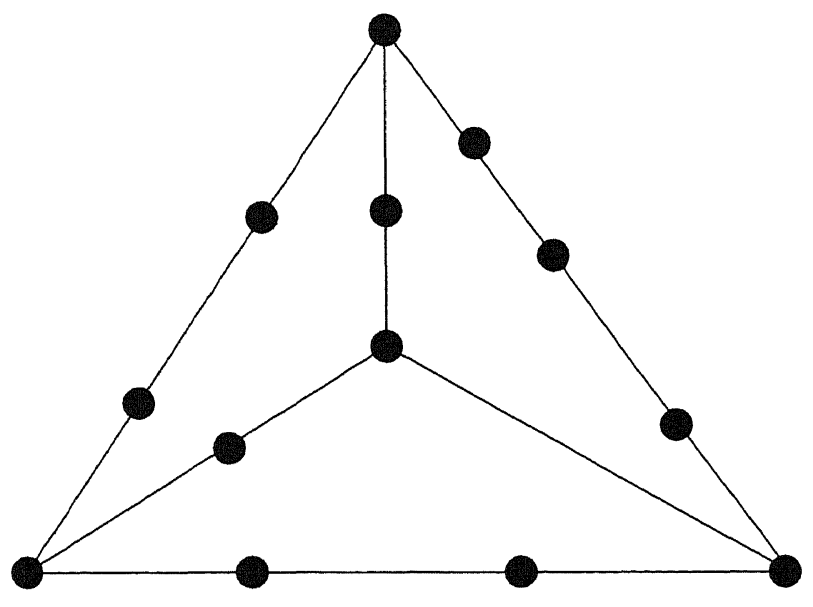

FIG. 5.3. A homeomorph of $K_{4}$.

at the end of section 2.2. A natural question concerns the complexity of the problem for graphs of order 2 .

The graphs of order 2 have been characterized in [28]. It is shown there that, up to a simple graph operation (clique-sum), they belong to two basic classes $\mathcal{G}_{1}$ and $\mathcal{G}_{2}$. All the graphs in $\mathcal{G}_{1}$ have minimum fill-in at most 3 ; hence, the problem is polynomially solvable for them (by Theorem 1.2). The graphs in class $\mathcal{G}_{2}$ are the generalized wheels of length 4 (and unrestricted fatness). Hence, if the psd matrix completion problem is polynomially solvable for generalized wheels of length 4 , then the same holds for all graphs of order 2 .

5.3. The matrix completion problem for graphs with no homeomorph of $\boldsymbol{K}_{\mathbf{4}}$. We now discuss the matrix completion problem for the class $\mathcal{H}$ consisting of the graphs containing no homeomorph of $K_{4}$ as a subgraph; a homeomorph of $K_{4}$ being obtained from $K_{4}$ by replacing its edges with paths; cf. Figure 5.3 for an example. (Graphs in $\mathcal{H}$ are also known as series parallel graphs.) Clearly, $\mathcal{H}$ contains all circuits. The case of circuits is certainly interesting to understand since circuits are the most simple nonchordal graphs.

Similarly to the chordal case, a condition characterizing existence of a psd completion is known for the graphs in $\mathcal{H}$. Namely, the following is shown in [25] (using a result of [7]). Given a graph $G=(V, E)$ in $\dot{H}$ and $a \in \mathbf{R}^{V \cup E}$ satisfying $a_{i}=1$ for all $i \in V$, then $a$ has a psd completion if and only if the scalars $x_{e}:=\frac{1}{\pi} \arccos a_{e}(e \in E)$ satisfy the inequalities

(5.2) $\sum_{e \in F} x_{e}-\sum_{e \in C \backslash F} x_{e} \leq|F|-1$ for all $F \subseteq C$ with $C$ circuit in $G,|F|$ odd.

Proposition 5.3 (see [6]). Given $x \in[0,1]^{E}$, one can test in polynomial time whether $x$ satisfies the linear system (5.2).

Proof. Consider the graph $\tilde{G}:=\left(V \cup V^{\prime}, \tilde{E}\right)$ where $V^{\prime}:=\left\{i^{\prime} \mid i \in V\right\}$ and $\tilde{E}$ consists of the pairs $i j, i^{\prime} j^{\prime}, i j^{\prime}, i^{\prime} j$ for $i j \in E$. Define $z \in \mathbf{R}^{\tilde{E}}$ by $z_{i j}=z_{i^{\prime} j^{\prime}}=x_{i j}$ and $z_{i^{\prime} j}=z_{i j^{\prime}}=1-x_{i j}$ for $i j \in E$. Then, it is easy to see that $x$ satisfies (5.2) if and only if $z(P) \geq 1$ for every path $P$ from $i$ to $i^{\prime}$ in $\tilde{G}$ and every $i \in V$. The result now follows as one can compute shortest paths in polynomial time. 
Therefore, problem $\left(\mathrm{P}_{s}\right)$ is polynomial time solvable in the real number model for graphs in $\mathcal{H}$. It is not clear how to extend this result to the bit model since the scalars $x_{e}:=\frac{1}{\pi} \arccos a_{e}$ are in general irrational and, thus, one encounters problems of numerical stability when trying to check whether (5.2) holds.

Moreover, there is a simple combinatorial algorithm (already briefly mentioned in [26]) permitting us to construct a psd completion in polynomial time in the real number model. Let $G=(V, E)$ be a graph in $\mathcal{H}$ and let $a \in \mathbf{R}^{V \cup E}$ be given satisfying $a_{i}=1$ for all $i \in V$. The algorithm performs the following steps.

1. Set $x_{e}:=\frac{1}{\pi} \arccos a_{e}$ for $e \in E$ and test whether $x$ satisfies (5.2). If not, one can conclude that $a$ has no psd completion. Otherwise, go to step 2.

2. Find a set $F$ of edges disjoint from $E$ for which the graph $H:=(V, E \cup F)$ is chordal and contains no homeomorph of $K_{4}$.

3. Find an extension $y \in[0,1]^{E \cup F}$ of $x$ satisfying the linear system (5.2) with respect to graph $H$.

4. Set $b_{e}:=\cos \left(\pi y_{e}\right)$ for $e \in E \cup F$ and $b_{i}:=1$ for $i \in V$. Then, $b$ is completable to a psd matrix (since $y$ satisfies (5.2) and $H$ has no homeomorph of $K_{4}$ ) and one can compute a psd completion $X$ of $b$ with the algorithm of section 3.2 (since $H$ is chordal). Then, $X$ is a completion of $a$.

All steps can be executed in polynomial time. This follows from earlier results for steps 1 and 4; for step 2 use a result of [41] and, for step 3, one can use an argument similar to the proof of Proposition 5.3. Namely, given $x \in[0,1]^{E}$ satisfying (5.2), in order to extend $x$ to $[0,1]^{E \cup\{e\}}$ in such a way that (5.2) remains valid with respect to $G+e$, one has to find a scalar $\alpha \in[0,1]$ satisfying $L_{1} \leq \alpha \leq L_{2}$, where

$$
\begin{gathered}
L_{1}:=\max _{C, F \mid e \in C \backslash F}(x(F)-x(C \backslash(F \cup\{e\}))-|F|+1), \\
L_{2}:=\min _{C, F \mid e \in F}(x(C \backslash F)-x(F \backslash e)+|F|-1) .
\end{gathered}
$$

We have $L_{1} \leq L_{2}$ (since $x$ satisfies (5.2)) and $L_{1} \leq 1, L_{2} \geq 0$ (since $x \in[0,1]^{E}$ ); thus, $\left[L_{1}, L_{2}\right] \cap[0,1] \neq \emptyset$. With the notation of the proof of Proposition 5.3, one finds that

$$
\begin{gathered}
L_{1}=1-\min \left(z(P) \mid P \text { is an } a b^{\prime} \text {-path in } \tilde{G}\right), \\
L_{2}=\min (z(P) \mid P \text { is an } a b \text {-path in } \tilde{G}) .
\end{gathered}
$$

Hence one can compute $\alpha$ in polytime. One can then determine the extension $y$ of $x$ to $H$ by iteratively applying this procedure.

The distance matrix completion problem for graphs in $\mathcal{H}$ can be treated in a similar manner. Indeed, given $G=(V, E)$ in $\mathcal{H}$ and $a \in \mathbf{R}_{+}^{E}$, set $x_{e}:=\sqrt{a}_{e}$ for $e \in E$. Then, $a$ is completable to a distance matrix if and only if $x$ satisfies the linear inequalities

$$
x_{e}-\sum_{f \in C \backslash e} x_{f} \leq 0 \text { for all circuits } C \text { in } G \text { and all } e \in C
$$

(cf. [27]). Again one can test in polynomial time whether $x \geq 0$ satisfies (5.3). (Simply, test for each edge $e=a b \in E$ whether $x_{e} \leq \min (x(P) \mid P$ is an $a b$-path in $G)$.) An algorithm analogous to the one exposed in the psd case permits us to construct a distance matrix completion. Therefore, we have shown the following theorem. 
THEOREM 5.4. One can construct a real psd (distance matrix) completion or decide that none exists in polynomial time in the real number model for the graphs containing no homeomorph of $K_{4}$.

It is an open question whether the above result extends to the bit model of computation, even for the simplest case of circuits.

Acknowledgments. We are grateful to A. Barvinok for providing us insight about Theorem 5.2, to L. Porkolab for bringing [24] to our attention, and to A. Schrijver for discussions about section 3. We also thank the referees for their careful reading and for their suggestions which helped us improve the presentation of the paper.

\section{REFERENCES}

[1] J. Agler, J. W. Helton, S. McCullough, And L. Rodman, Positive semidefinite matrices with a given sparsity pattern, Linear Algebra Appl., 107 (1988), pp. 101-149.

[2] A. Y. AlfaKiH, A. KhaNDANI. AND H. WolKowICZ, Solving Euclidean distance matrix completion problems via semidefinite programming, Comput. Optim. Appl., 12 (1998), pp. 13-30.

[3] F. AlIZADEH, Interior point methods in semidefinite programming with applications in combinatorial optimization, SIAM J. Optim., 5 (1995), pp. 13-51.

[4] M. BAKONYI AND C. R. JoHnSON, The Euclidean distance matrix completion problem, SIAM J. Matrix Anal. Appl., 16 (1995), pp. 646-654.

[5] M. BAKONYI AND G. NAEVDAL, On the matrix completion method for multidimensional moment problems, Acta Sci. Math. (Szeged), 64 (1998), pp. 547-558.

[6] F. Barahona and A. R. Mahjoub, On the cut polytope, Math. Programming, 36 (1986), pp. 157-173.

[7] W. BARRETt, C. R. Johnson, AND P. TARAzAGA, The real positive definite completion problem for a simple cycle, Linear Algebra Appl., 192 (1993), pp. 3-31.

[8] A. I. BARVINOK, Feasibility testing for systems of real quadratic equations, Discrete Comput. Geom., 10 (1993), pp. 1-13.

[9] A. I. BARVINOK, personal communication, 1998.

[10] L. Blum, M. Shub, AND S. SMale, On a theory of computation and complexity over the real numbers: NP-completeness, recursive functions and universal machines, Bull. Amer. Math. Soc., 21 (1989), pp. 1-46.

[11] G. M. CRIPPEn AND T. F. HAVEl, Distance Geometry and Molecular Conformation, Research Studies Press, Taunton, Somerset, England, 1988.

[12] H. DYM AND I. GoHBERG, Extensions of band matrices with band inverses, Linear Algebra Appl., 36 (1981), pp. 1-24.

[13] M. R. Garey and D. S. Johnson, Computers and Intractability: A Guide to the Theory of NP-Completeness, W. H. Freeman and Company, New York, 1979.

[14] M. C. Golumbic, Algorithmic Theory and Perfect Graphs, Academic Press, New York, 1980.

[15] R. Grone, C. R. Johnson, E. M. SÁ, and H. Wolkowicz, Positive definite completions of partial Hermitian matrices, Linear Algebra Appl., 58 (1984), pp. 109-124.

[16] M. Grötschel, L. LovÁsz, AND A. SCHriJver, Geometric Algorithms and Combinatorial Optimization, Springer-Verlag, Berlin, 1988.

[17] C. Helmberg, F. Rendl, R. J. Vanderbei, and H. Wolkowicz, An interior-point method for semidefinite programming, SIAM J. Optim., 6 (1996), pp. 342-361.

[18] B. HENDRICKSON, The molecule problem: Exploiting structure in global optimization, SIAM J, Optim., 5 (1995), pp. 835-857.

[19] C. R. Johnson, Matrix completion problems: A survey, in Matrix Theory and Applications 40 , Proc. Sympos. Appl. Math., C. R. Johnson, ed., AMS, Providence, RI, 1990, pp. 171-198.

[20] C. R. Johnson, B. Kroschel, And H. Wolkowicz, An interior-point method for approximate positive semidefinite completions, Comput. Optim. Appl., 9 (1998), pp. 175-190.

[21] C. R. Johnson AND P. TARAzAGa, Connections between the real positive semidefinite and distance matrix completion problems, Linear Algebra Appl., 223/224 (1995), pp. 375-391.

[22] N. Karmarkar, A new polynomial-time algorithm for linear programming, Combinatorica, 4 (1984), pp. 373-395.

[23] L. KHACHIYAN, A polynomial algorithm in linear programming, Soviet Mathematics Doklady, 20 (1979), pp. 191-194. 
[24] L. KhachIYAN AND L. PORKOlab, Computing integral points in convex semi-algebraic sets, in 38th Annual IEEE Symposium on Foundations of Computer Science, Miami, FL, 1997, pp. $162-171$

[25] M. LAURENT, The real positive semidefinite completion problem for series-parallel graphs, Linear Algebra Appl., 252 (1997), pp. 347-366.

[26] M. LAurent, Cuts, matrix completions, and graph rigidity, Math. Programming, 79 (1997), pp. $255-283$.

[27] M. LAURENT, A connection between positive semidefinite and Euclidean distance matrix completion problems, Linear Algebra Appl., 273 (1998), pp. 9-22.

[28] M. LAURENT, On the order of a graph and its deficiency in chordality, Combinatorica, to appear.

[29] J. DE LEEUW AND W. HeISER, Theory of multidimensional scaling, in Handbook of Statistics, Vol. 2, P. R. Krishnaiah and L. N. Kanal, eds., North Holland, 1982, pp. 285-316.

[30] H. W. LENSTRA, JR., Integer programming with a fixed number of variables, Math. Oper. Res., 8 (1983), pp. $538-548$.

[31] J. J. MORÉ AND Z. WU, Distance geometry optimization for protein structures, J. Global Optim., 15 (1999), pp. 219-234.

[32] Y. E. Nesterov And A. S. Nemirovsky, Interior Point Polynomial Algorithms in Convex Programming: Theory and Algorithms, SIAM, Philadelphia, 1994.

[33] L. Porkolab and L. KhachiYan, On the complexity of semidefinite programs, J. Global Optim., 10 (1997), pp. 351-365.

[34] L. PORKOLAB, private communication, 2000.

[35] M. V. RamanA, An exact duality theory for semidefinite programming and its complexity implications, Math. Programming, 77 (1997), pp. 129-162.

[36] D. J. Rose, R. E. TARjan, ANd G. S. Lueker, Algorithmic aspects of vertex elimination on graphs, SIAM J. Comput., 5 (1976), pp. 266-283.

[37] I. J. Schoenberg, Remarks to M. Fréchet's article "Sur la définition axiomatique d'une classe d'espaces vectoriels distanciés applicables vectoriellement sur l'espace de Hilbert," Ann. of Math., 36 (1935), pp. 724-732.

[38] A. SCHRIJVER, Theory of Linear and Integer Programming, John Wiley and Sons, New York, 1986.

[39] R. E. TARJAN, Decomposition by clique separators, Discrete Math., 55 (1985), pp. 221-232.

[40] L. VAndenberghe And S. Boyd, Semidefinite programming, SIAM Rev., 38 (1996), pp. 49-95.

[41] J. A. WALD AND C. J. Colbourn, Steiner trees, partial 2-trees and minimum IFI networks, Networks, 13 (1983), pp. 159-167.

[42] H. Wolkowicz, R. Saigal, and L. Vandenberghe, eds., Handbook of Semidefinite Programming: Theory, Algorithms, and Applications, Kluwer Academic Publishers, Dordrecht, The Netherlands, 2000.

[43] S. J. Wright, Primal-Dual Interior-Point Methods, SIAM, Philadelphia, 1996.

[44] M. YanNaKaKIS, Computing the minimum fill-in is NP-complete, SIAM J. Algebraic Discrete Methods, 2 (1981), pp. 77-79. 